\title{
Rotation does not Enhance Mixing in the Mixmaster Universe ${ }^{\star}$
}

\author{
RichARD A. MATZNER \\ Department of Physics, University of Texas, Austin, Texas \\ D. M. CHITRE
}

Department of Physics and Astronomy, University of Maryland, College Park, Maryland

Received March 3, 1971

\begin{abstract}
We investigate closed rotating cosmologies to determine if rotation leads to an enhancement of causal mixing proposed by Misner to guarantee the homogeneity of such models. We conclude that rotation cannot lead to significantly more efficient mixing than occurs in non-rotating models. Since arguments presented by Doroshkevich and Novikov and calculations made by Chitre give very small probability of mixing in nonrotating models, we therefore conclude that a plausible explanation of the homogeneity of the universe cannot be found within the framework of classical General Relativity. Such an explanation may lie in quantum effects on mixing near the singularity.
\end{abstract}

\section{Introduction}

We consider here the phenomenon of "causal mixing", first postulated by Misner (1969a) for the Mixmaster universe (a closed non-rotating model). At any fixed time or volume epoch $\Omega$ near the singularity, the Mixmaster universe can be specified by initial conditions $\beta_{+}, \beta_{-}$(shape anisotropy) and $k_{+}, h_{-}$(expansion rate anisotropy). Chitre (1970) has shown that for certain subsets of initial conditions some null-geodesics will circumnavigate the corresponding universe and that a probability for this can be computed since the Einstein equations lead to a natural measure on initial conditions $\left(\beta_{ \pm}, \beta_{ \pm}\right)$. Doroshkevich and Novikov (1970) and Chitre (1971) find that the probability of removing horizons in any one direction is very low. That is, the possibility exists, but the probability of such behavior is quite small.

In this paper we discuss models with rotation. The basic idea is that in such models the rotation may move the mixing direction so that effective mixing in many directions is possible. We shall show that rotation leads to slightly more efficient mixing, but the improvement is in no

* Supported in part by NSF Grant No. GP-20033, by NASA Grant No. NGR-21-002010, and by the Center for Theoretical Physics, University of Maryland.

13 Commun. math. Phys, Vol. 22 
case particularly striking. We conclude that rotation does not make the removal of horizons in these models significantly more probable than it is in non-rotating models. Rotation does not save the Mixmaster universe.

The metric describing a rotating closed cosmology of Bianchi type IX can be written (Ryan, 1970)

$$
d s^{2}=-d t^{2}+R^{2}(t) e_{i j}^{2 \beta(t)} \sigma^{i} \sigma^{j},
$$

where the time independent covariant vectors $\sigma^{i}$ are invariant under the action of the group of motions of Bianchi type IX. Hence, they obey the curl relation

$$
d \sigma^{i}=\varepsilon_{i j k} \sigma^{i} \wedge \sigma^{k} .
$$

The function $R(t)$ describes the size of the universe, and the matrix $e^{2 \beta(t)}$ gives the anisotropy of the model. (We take $\beta_{i i}=0$ so $e^{2 \beta}$ has unit determinant.) A parametrization of $\beta_{i j}$ can be given in terms of the following set of variables: $\left(\beta_{+}, \beta_{-}, \varphi, \theta, \psi\right)$. Here $\beta_{+}$and $\beta_{-}$are two linear combinations of the eigenvalues of $\beta_{i j}$ (Misner, 1968):

$$
\beta_{+}=-\frac{1}{2} \beta_{3}, \quad \beta_{-}=(2 \sqrt{3})^{-1}\left(\beta_{1}-\beta_{2}\right),
$$

where $\beta_{1}, \beta_{2}, \beta_{3}$ are the eigenvalues of $\beta$ in any order $\left(\beta_{1}+\beta_{2}+\beta_{3}=0\right)$. The angles $\varphi, \theta, \psi$ are the Euler angles of the rotation relating $\beta_{i j}$ to the diagonal matrix with the same eigenvalues. $\left(\beta=0^{T} b 0\right.$ with $b$ diagonal and $0=\mathscr{R}_{z}(\varphi) \mathscr{R}_{x}(\theta) \mathscr{R}_{z}(\psi)$; see Ryan, 1970 or Matzner, 1971.)

We shall follow Misner $(1967,1968)$ and take one of the dynamic equations of the system to be the $T_{00}=G_{00}$ equation; we will at the same time relegate the $T_{k}^{k}=G_{k}^{k}$ (spatial trace) equation to secondary status. The $T_{00}=G_{00}$ equation reads

Here

$$
3(\dot{R} / R)^{2}=\frac{1}{2} \sigma_{i j} \sigma_{i j}+\varrho_{g}\left(V_{g}-1\right)+T_{00} .
$$

$$
\sigma_{i j}=\frac{1}{2}\left[\left(e^{\beta}\right)^{\cdot} e^{-\beta}+e^{-\beta}\left(e^{\beta}\right)^{\cdot}\right]_{i j} .
$$

(The dot means time derivative.) The quantities $\varrho_{g}$ and $V_{g}$ are defined

$$
\varrho_{g}=\frac{3}{4} R^{-2}, \quad V_{g}=\frac{1}{3} \operatorname{trace}\left(e^{4 \beta}-2 e^{-2 \beta}+1\right) .
$$

Because of the symmetry of $V_{g}(\beta)$ under interchange of the labeling of eigenvalues, $V_{q}$ has triangularly symmetric equipotentials in the $\beta_{+} \beta_{-}$ plane. These approximate straight-sided equilateral triangles for large $V_{g}$. One wall of each equipotential triangle cuts the negative $\beta_{+}$axis at right angles, and in the triangular section including the negative $\beta_{+}$axis,

$$
V_{g} \sim \frac{1}{3} e^{-8 \beta+}
$$


In the corners of the triangle, the equipotentials curve outward to infinity so the potential is bounded, $V_{g}<1$, along the $\beta_{+}>0$ axis and the two triangularly symmetric directions. (See Misner, 1969a, b; Matzner, Shepley, Warren, 1970: "MSW;" Ryan, 1970.)

Still following Misner (1967), we obtain the

$$
T_{j}^{i}-\frac{1}{3} \delta_{j}^{i} T_{k}^{k}=G_{j}^{i}-\frac{1}{3} \delta_{j}^{i} G_{k}^{k}
$$

equations by the variation $\delta^{\prime} \beta_{i j}$ in the Lagrangian

$$
\mathscr{L}=R^{3}\left[\frac{1}{2} \sigma_{i j} \sigma_{i j}-\varrho_{g}\left(V_{g}-1\right)-T_{00}\right] .
$$

(Specifically, one varies $e_{i j}^{2 \beta}$; the coefficients of $\delta^{\prime} \beta \equiv e^{-\beta} \delta\left(e^{2 \beta}\right) e^{-\beta}$ give the traceless spatial components of the field equations in the orthonormal frame $\left\{d t, \omega^{i}=R^{-1} e_{i j}^{-\beta} \sigma^{j}\right\}$.) Misner $(1967,1968)$ has shown that the Einstein tensor components are correctly given by this variational principle if one treats $R$ as an externally given function of time. For fluids and for general models containing collisionless radiation, Matzner (1970, 1971) has shown that $R^{3} T_{00}$ is indeed the correct matter Lagrangian. [If $T_{00}$ is due to a fluid, certain conservation laws also have to be respected; see Matzner (1970).] We find it useful to work directly in the "metric coordinates" $\beta_{Y}=\left(\beta_{+}, \beta_{-}, \varphi, \theta, \psi\right)$. We begin by translating the Lagrangian into this formulation (Ryan, 1970; Matzner, 1971):

$$
\begin{aligned}
\mathscr{L}= & R^{3}\left\{3 \dot{\beta}_{+}^{2}+3 \dot{\beta}_{-}^{2}+\sinh ^{2}(2 \sqrt{3}) \beta_{-}\left(\frac{\sigma^{3}}{d t}\right)^{2}\right. \\
& +\sinh ^{2}\left[3 \beta_{+}-(\sqrt{3}) \beta_{-}\right]\left(\frac{\sigma^{1}}{d t}\right)^{2} \\
& \left.+\sinh ^{2}\left[3 \beta_{+}+(\sqrt{3}) \beta_{-}\right]\left(\frac{\sigma^{2}}{d t}\right)^{2}-\varrho_{g}\left[V_{g}\left(\beta_{+}, \beta_{-}\right)-1\right]-T_{00}\right\},
\end{aligned}
$$

where any explicit appearances of the metric in $T_{00}$ have been parametrized in terms of the $\beta_{Y}$. (We assume no derivatives of the metric appear in $T_{00}$, and we also assume that other parameters entering $T_{00}$ are treated as explicitly given functions of $t$, as is $R$.) The $\sigma^{i}, i=1,2,3$ are a set of inexact differentials:

$$
\begin{aligned}
& \sigma^{1}=-\cos \varphi d \theta-\sin \varphi \sin \theta d \psi \\
& \sigma^{2}=\cos \varphi \sin \theta d \psi-\sin \varphi d \theta, \\
& \sigma^{3}=d \varphi+\cos \theta d \psi
\end{aligned}
$$

[The $\sigma^{i}$ here may be taken as a coordinate realization of the $\sigma^{i}$ introduced in Eq. (1.1) since they obey $d \sigma^{1}=\sigma^{2} \wedge \sigma^{3}$ et. cyc.] 
During the large anisotropy evolution of a universe model, the influence of $T_{00}$ on the anisotropy can be described by introducing potentials due to the matter; one has (Matzner, 1970, 1971)

$$
T_{00} \simeq \mathscr{A} R^{-4} V_{\text {matter }}\left(\beta_{v} t\right),
$$

where $\mathscr{A}$ is a constant. The "walls" defined by $V_{\text {matter }}$ (i.e., the instantaneous locus of turning points) can be shown to move in such a way that collisions of the system point with them are virtually impossible. One "bounce" off the wall is the maximum allowed in the large anisotropy region. (See Misner, 1968; Matzner, 1970, 1971; or Ryan, 1970.) Hence, so far as the dynamical evolution is concerned, we may neglect $T_{00}$ in the Lagrangian during the large anisotropy regime. We wish to discuss the Mixmaster phenomenon which perforce occurs during the large anisotropy regime; hence, we drop $T_{00}$ from now on.

\section{Critique of the Non-Rotating Mixmaster}

In the absence of momentum currents $T_{0 i}$, the $T_{0 i}=G_{0 i}$ equations require a diagonal metric, as is well known. One then takes $\varphi=\psi=\theta=0$, and $\beta_{+}, \beta_{-}$are the only two dynamic variables necessary to describe the solution. A general feature of such models is the following relation (Misner, 1967, 1968; Ryan, 1970; MSW, 1970). Define

$$
\Omega=\ln \left(R_{0} / R\right) \quad\left(R_{0} \text { constant }\right) .
$$

Then

$$
\left(d \beta_{+} / d \Omega\right)^{2}+\left(d \beta_{-} / d \Omega\right)^{2} \approx 1 .
$$

This equation also follows in rotating models from Eq. (1.4) (Ryan, 1970; MSW, 1970).

Misner (1969a) has described the Mixmaster behavior of nonrotating models described by (1.1). Essentially, if $\beta_{+}, \beta_{-}$describing the metric moves exactly in the direction of one of the corners of $V_{g}$, then (a) such a motion can persist for a very long time since the potential $V_{g}$ has channels in the corner directions and is bounded in those directions, and (b) if the motion persists sufficiently long in this manner, a photon has an opportunity to completely circumnavigate the universe in the direction where the metric coefficient is smallest (the 3 -axis if the system point is in the channel $\beta_{+} \gg 1, \beta_{-} \sim 0$ ). Chitre (1970) has shown that there exists a set of initial conditions (of non-zero measure) for which the above Kasner-like behavior persists long enough for the horizon to vanish.

Recently, however, Chitre (1971) has shown that the probability of mixing is quite small in any particular model. Previously, Doroshkevich and Novikov (1970) pointed out that it is extremely improbable that the 
system point move in a way which removes horizons unless it enters the channel during the small anisotropy phase of the model. (Recall $\Omega$ increases toward the singularity; we will use this inverted time sense throughout the paper.) It is thus very improbable that the system point will enter a second channel after emerging from one, and it is necessary that horizons be removed in all directions for really effective mixing to take place; hence, three "corner runs" are required. We will not explicitly obtain this result for the non-rotating case, but because of the close similarity in their Mixmaster behavior of the non-rotating models and the non-tumbling, rotating models (defined in Section IV), we can demonstrate this fact by quoting the results from Section IV. Because of the small $\beta_{-}$motion in a region where the potential is approximately harmonic [see Eq. (4.1)], there are small oscillations in $\beta_{\text {- with frequency }}$

$$
\omega_{-}=4 \sqrt{3} e^{2\left(\beta_{+}-\beta_{c}\right)} .
$$

The quantity $\beta_{c}$ is defined as the distance from the origin in the $\beta_{+}, \beta_{-}$ plane to the point where two sides of the triangular equipotential of $V_{g}$ intersect if extended in straight lines. Now $\beta_{c}$ moves with $d \beta_{c} / d \Omega \simeq 1$ [Eqs. (4.6) and (4.7) below], so if the system point starts "chasing the corner" at any sizable value of $\Omega$, say $\Omega_{0}, \omega_{-}$will be extremely small; $\omega_{-} \propto e^{-2 \Omega_{0}}$.

In a suitably oriented coordinate system $x, y, z$ on the model, the equation for the $z$ coordinate of a photon is (Chitre, 1970; see also Section V)

$$
d z / d \Omega=2 \sqrt{3} e^{2\left(\beta_{+}-\beta_{c}\right)} .
$$

The model describes a closed universe, so $z$ is interpreted as an angular variable and $\Delta z=4 \pi$ describes a complete circumnavigation of the universe. For the infinitesimal $\beta_{-}$analysis when the system point moves in the channel, $\omega_{-}$is an adiabatic invariant (Misner, 1969a). Eq. (2.4) then shows that circumnavigation will eventually occur after precisely four oscillations in $\beta_{-}$. If, however, the system is moving at a finite angle $\lambda$ to the channel direction, the system point $\beta_{+}$lags behind $\beta_{c}$, and one has in fact

while

$$
\beta_{+} \simeq \beta_{+0}+\Omega \cos \lambda
$$

$$
\beta_{c} \simeq \beta_{c 0}+\Omega \text {, }
$$

where $\beta_{+0}$ and $\beta_{c 0}$ are constants, and we have introduced the $\left|\frac{d \beta}{d \Omega}\right| \simeq 1$ and $\left|\frac{d \beta_{c}}{d \Omega}\right| \simeq 1$ behavior characteristic of free motion. But then it is clear 
that $\int d z$ converges; one has

$$
\Delta z \equiv \int_{0}^{\infty} \frac{d z}{d \Omega} d \Omega=\frac{\sqrt{3} e^{2\left(\beta+0-\beta_{c 0}\right)}}{1-\cos \lambda} .
$$

Hence, $\Delta z \geqq 4 \pi$ requires

$$
|\lambda| \approx\left(3^{1 / 2} / 2 \pi\right)^{1 / 2} e^{\left(\beta+0-\beta_{c 0}\right)},
$$

when the right-hand side is small.

The meaning of Eq. (2.6) is that, if the system starts moving in the direction of the corner but is far from the corner, complete circumnavigation (and hence mixing) can occur only if the system direction makes an angle $|\lambda|$ with the corner direction which satisfies (2.6).

We have a Hamiltonian system [see Eq. (3.4) below], and the parameter $\lambda$ gives the direction of the momentum in phase space. All directions are a priori equally probable. Taking into account the 3-fold symmetry and the fact that it is $\lambda^{2}$ which enters, the probability of approaching one of the channels within an angle $|\lambda|$ is $P=6|\lambda| / 2 \pi$; hence, the probability of satisfying Eq. (2.6) is

$$
P \simeq 3^{1 / 4} 6 e^{\left(\beta+0-\beta_{c 0}\right)}(2 \pi)^{-3 / 2} .
$$

Suppose we have a corner run which began when $\beta_{c}=\beta_{c 0}$ and which persists just long enough for mixing to occur, i.e., for an interval $\Delta \Omega=8 \pi \omega_{-}^{-1}$. It is important to realize that $\beta_{c 0}$ must be of order $\sim 3$ or 5 since otherwise the large anisotropy discussion (particularly $d \beta / d \Omega \simeq 1$ ) will not hold. After the completion of this first corner run, the system must move back into the center of the potential before beginning a second such corner run. The minimum time for this to occur is $\Delta \Omega=\beta_{+f}$, where $\beta_{+f}$ is the value of $\beta_{+}$in the corner at the completion of the run: $\beta_{+f} \simeq 8 \pi \omega_{-0}^{-1}$. Hence, $\Delta \Omega$ for the completion of one run and the preparation for a second is

$$
\Delta \Omega \widetilde{>} 16 \pi \omega_{-0}^{-1},
$$

where $\omega_{-0}$ is the frequency appropriate to the first corner run.

The value of the corner distance for the second run, $\beta_{c 1}$, is thus $\beta_{c 1} \geqq \beta_{c 0}+16 \pi \omega_{-0}^{-1}$. Hence,

$$
\omega_{-1}=4 \sqrt{3} e^{-2 \beta_{c 1}} \leqq 4 \sqrt{3} e^{-2\left(\beta_{c 0}+16 \pi \omega_{-1}^{-1}\right)} .
$$

Because the probability of entering a second corner is $\propto \omega_{-1}^{1 / 2}$ [Eq. (2.7)], we see that if $\omega_{-0}$ is small (i.e., if the first corner run did not begin very near the maximum of expansion), the probability for a second and third mixing run are very small indeed. More precise calculations verifying these estimates have been presented elsewhere (Chitre, 1971); this argument was first presented by Doroshkevich and Novikov (1970). 
It has been shown in prior investigations (MSW; Ryan, 1970; Matzner, 1970; Matzner, 1971) that rotation affects the dynamics of $\beta_{+}$and $\beta_{-}$very little during most phases of the evolution of a model. Hence, we accept for our rotating models the above estimates from the non-rotating models. We shall, however, suppose that one corner run does occur and look for ways to make this single corner run effective. The mechanism which presents itself as a candidate to improve the mixing efficiency is the rotation. As has been mentioned by several authors (MSW; Ryan, 1970; Matzner, 1970), rotation can turn the direction of the mixing axis and thereby enhance the mixing so that perhaps only one corner run will do, and the improbable second and third ones are not needed. We shall see that in fact the enhancement due to rotation is small.

\section{The Rotating Type IX Models}

With $R^{3} T_{00}$ deleted in $\mathscr{L}$, it is apparent that $\psi$ is a cyclic variable; $h_{\psi}=$ constant $\left(h_{\psi}\right.$ is the momentum conjugate to $\left.\psi\right)$ is a first integral of the system. In addition, $\theta$ and $\varphi$ appear only in the $\sigma^{i} / d t$ terms, and these have exactly the form of the angular velocity of an asymmetrical rotator expressed in the "body frame". (See Goldstein, 1959, p. 134, but beware of differences in Euler angle definition.) Hence, we might expect that the total angular momentum $\not^{2}$ (formed in the usual way from the Eulerangle conjugate momenta) to be conserved; it is easy to verify that this is the case:

$$
\not h^{2} \equiv h_{\varphi}^{2}+h_{\theta}^{2}+\left(h_{\psi}-\cos \theta h_{\varphi}\right)^{2}(\sin \theta)^{2}
$$

is a constant of the motion for this system. Here $h_{\theta}$ and $h_{\varphi}$ are the momenta conjugate to $\theta$ and $\varphi$.

It can be shown that the initial values of $T_{0 i}$ determine an initial value for $h^{2}$; in fact, the $T_{0 i}$ are certain definite functions of $h_{\varphi}, h_{\theta}$, and $h_{\psi}$ and of $R$ and $\beta_{Y}$. Since, as we have noted, the matter terms affect the dynamics very little, the equations (which we shall not write here) relating $T_{0 i}$ and the $k_{Y}$ should be read, subsequent to the initial instant, as giving $T_{0 i}$ in terms of $\mu_{Y}$.

It is useful for the analysis of the Mixmaster behavior to introduce the ADM Hamiltonian formulation (Arnowitt, Deser, Misner, 1962; Misner, 1969b). This can be obtained by applying the ADM procedure to the Bianchi type IX metric, with $T_{00}=0$ to be consistent with our conclusion that $T_{00}$ does not affect the dynamics in the large anisotropy regime. Alternately, one can introduce $l \equiv(d t / d \Omega) \mathscr{L}$. Then the action principle becomes $\delta \int l d \Omega=0$. The momenta $\beta_{Y}$ conjugate to $\beta_{Y}$ are unchanged by this transformation. Introduce the Hamiltonian associated 
with $l$ :

$$
h=\frac{d \beta_{Y}}{d \Omega} p_{Y}-l
$$

Notice from Eqs. (1.4) and (2.1) that

$$
\bar{h}=3(d \Omega / d t) R^{3},
$$

where $\bar{h}$ denotes the numerical value of $h$; and $\frac{1}{2} \bar{h}^{-1}$ is a factor on the right-hand side of the definition of $h$, Eq. (3.2). One can then show that the equations of motion obtained from $h$ are identical to those obtained from the Hamiltonian system $\not_{Y}, \beta_{Y}, H$, where (Ryan, 1970)

$$
\begin{aligned}
H^{2}= & \not_{+}^{2}+\not_{-}^{2}+\frac{3 \not_{\varphi}^{2}}{\sinh ^{2}(2 \sqrt{3}) \beta_{-}} \\
& +3 \frac{\left\{\not_{\theta} \cos \varphi+(\sin \theta)^{-1}\left[\not_{\psi}-\not_{\varphi} \cos \theta\right] \sin \varphi\right\}^{2}}{\sinh ^{2}\left[3 \beta_{+}-(\sqrt{3}) \beta_{-}\right]} \\
& +3 \frac{\left\{\not_{\theta} \sin \varphi-(\sin \theta)^{-1}\left[\not_{\psi}-\not_{\varphi} \cos \theta\right] \cos \varphi\right\}^{2}}{\sinh ^{2}\left[3 \beta_{+}+(\sqrt{3}) \beta_{-}\right]} \\
& +12 \varrho_{g}\left(V_{g}-1\right) R^{6} .
\end{aligned}
$$

$H$ is the ADM Hamiltonian. We note that $\bar{H}=2 \bar{h}$. (We shall henceforth drop the bar notation for the value of $H$.) We repeat that the $k_{Y}$ here have the same numerical value as the $h_{Y}$ of the Lagrangian formulation of Eq. (1.7), and hence, our statements about the constancy of $k_{\psi}$ and of $h^{2}$ hold here also. [We did not deal immediately with the ADM formulation because there is some difficulty in expressing the general $T_{\mu \nu}$ in terms appropriate to the method; Ryan (1970) has discussed this problem.]

\section{The Non-tumbling Mixmaster}

In a special case of the rotating type IX models, the principal axes of the metric rotate about a single fixed axis, which can be taken to be the 3 -axis. The angles $\psi$ and $\theta$ are permanently zero and only $\varphi$ changes. Neither the axis of rotation nor the direction of matter flow changes so these models have been called non-tumbling in contrast to the general rotating (tumbling) case where $\varphi, \theta$, and $\psi$ all change (MSW).

In such a model, there are two possible types of Mixmaster behavior. One of the channel directions $\left(\beta_{+}>0, \beta_{-} \simeq 0\right.$ ) corresponds to a shortened axis which is the same as the rotation axis. The other channel directions correspond to a shortened axis which is not the same as the rotation axis. The two situations then can be specified by saying whether the 
mixing and rotation axis coincide (first case) or are different. In the second case, the rotation turns the mixing direction, and this turning mixing direction may lead to more effective mixing. We shall see below that there is in fact only a slight improvement.

We first treat the case with mixing and rotation axes parallel; the second case is taken up in Section VI. We suppose that the anisotropy parameter $\beta_{-}$is small, and $h_{\varphi}$ is non-zero but small. The Mixmaster behavior consists of motion which has the system point moving in the $\beta_{+} \beta_{-}$plane toward the corner channel of $V_{g}$ (here taken to be the channel along the $\beta_{+}>0$ axis). Along this axis the dominant terms in $V_{g}$ for $\beta_{+} \gg 1$ are (Misner, 1969)

$$
V_{g} \simeq 1+16 \beta_{-}^{2} e^{4 \beta+}
$$

With this approximation, one may now look for solutions in which $H \simeq p_{+}$; this then implies $d \beta_{+} / d \Omega \simeq 1$. Hence, the potential term in $H$ has the form $12 \varrho_{g}\left(V_{g}-1\right) R^{6}=(3 \times 4)^{2} R_{0}^{4} e^{4 \beta_{+}-4 \Omega} \beta_{-}^{2}$, and the coefficient of $\beta_{-}^{2}$ is approximately a constant. Based on this fact, Misner (1969) discussed the case with $h_{\varphi}=0$ and $\beta_{-}$and $\beta_{-}$small. In the non-tumbling model, we see that (at least for small $\beta_{-}$) $\not_{\varphi}$ enters exactly as would the centrifugal terms in ordinary Newtonian mechanics; the term involving $h_{\varphi}$ in $H^{2}$ is (Ryan, 1970)

$$
\frac{3 \not_{\varphi}^{2}}{\sinh ^{2} 2 \sqrt{3} \beta_{-}} \simeq \frac{\not_{\varphi}^{2}}{4 \beta_{-}^{2}} .
$$

The Hamiltonian equations for $\beta_{-}$and $\varphi$ then resemble those for a 2-dimensional harmonic oscillator expressed in cylindrical coordinates. The motion can be decomposed into two circular modes with $\beta_{-}=$constant and opposite values of $h_{\varphi}$. These can then be superposed to give the resultant (in general, elliptical) orbit in terms of $\beta_{-}, \varphi$. We briefly sketch the circular mode behavior.

In this case, $\not_{-}=0$, and the $p_{\varphi}$ terms balance the potential terms in the Hamiltonian equation for $\beta_{-}$. Assuming $\beta_{-}$is small, one finds

$$
\beta_{-}^{2}=\frac{\not_{\varphi} e^{-2\left(\beta_{+}-\Omega\right)}}{[2 \times 3 \times 4] R_{0}^{2}} .
$$

Here $\beta_{+}-\Omega$ is a slowly varying quantity as we mentioned above.

So long as $\beta_{-}$is small and so long as $e^{-\left(\beta_{+}-\Omega\right)}$ does not change much in one period of the $\varphi$ motion, Eq. (4.3) holds and gives the adiabatic change in $\beta_{-}^{2}$ with the slow change in $\left(\beta_{+}-\Omega\right)$. Notice that the potential remains central in the $\beta_{r} \beta_{d}$ plane, so $\beta_{\varphi}$ is exactly a constant of the motion, regardless of whether the adiabatic approximation holds. Eq. (4.3) is exactly the behavior predicted for the average behavior of $\beta_{-}^{2}$ in Misner's (1969) non-rotating adiabatic discussion. 
We may also obtain the equation of motion for $\varphi$ :

$$
\frac{d \varphi}{d \Omega}=\frac{\partial H}{\partial \not_{\varphi}} \simeq \frac{1}{H} \frac{\not \mu_{\varphi}}{4 \beta_{-}^{2}} .
$$

From Eq. (4.3), we have

$$
\omega_{-} \equiv 2 \frac{d \varphi}{d \Omega}=\frac{12}{H} e^{2\left(\beta_{+}-\Omega\right)} R_{0}^{2} .
$$

Since $\varphi$ enters the non-tumbling metric as $2 \varphi$ [Eqs. (5.6) below], this frequency is in agreement with Misner's previous calculation (1969) for the $\beta_{-}$oscillation frequency.

We may compute the equation of motion for $\beta_{+}$:

$$
\begin{aligned}
\frac{d \beta_{+}}{d \Omega}=\frac{\partial H}{\partial p_{+}}=\frac{h_{+}}{H} & \simeq 1-\frac{1}{2}\left[\frac{\not_{\varphi}^{2}}{4 \beta_{-}^{2}} \frac{1}{\not_{+}^{2}}+\frac{(12)^{2} R_{0}^{4}}{\not_{+}^{2}} e^{4\left(\beta_{+}-\Omega\right)} \beta_{-}^{2}\right] \\
& \simeq 1-\not_{+}^{-2}(12)^{2} R_{0}^{4} e^{4\left(\beta_{+}-\Omega\right)} \beta_{-}^{2} .
\end{aligned}
$$

So long as $\beta_{-}$is small, $d \beta_{+} / d \Omega$ remains very close to unity. Note, however, that it differs from unity by terms of order $\beta_{-}^{2}$.

We introduce the concept of the walls associated with $V_{g}$ by defining them to be the locus of points $\beta_{ \pm}^{\prime}$, where $12 \varrho_{g}\left[V_{g}\left(\beta_{+}^{\prime}, \beta_{-}^{\prime}\right)-1\right] R^{6}$ equals the instantaneous value of $H^{2}$. Using the approximate form for $V_{g}$ (away from one of the corner directions) given by Eq. (1.5), the distance from the origin of the $\beta_{+} \beta_{-}$plane to one of the walls of the potential is given by $\beta_{w}$, where

$$
\beta_{w}=\frac{1}{8} \ln \left[\frac{H^{2}}{3 R_{0}^{4}} e^{+4 \Omega}\right]=\frac{1}{2} \Omega+\frac{1}{8} \ln \left(\frac{H^{2}}{3 R_{0}^{4}}\right) .
$$

Because of the equilateral triangular symmetry of the potential $V_{g}$, if the sides were extended as straight lines, they would meet in corners making $60^{\circ}$ angles. The distance $\beta_{c}$ from such a corner to the origin is

$$
\beta_{c}=2 \beta_{w} .
$$

Of course, the actual equipotential contour curves outward to form the channel, but $\beta_{c}$ is a useful quantity. We note that $e^{2 \beta_{c}}=e^{+2 \Omega}(H / \sqrt{3}) R_{0}^{-2}$, so

$$
\omega_{-}=4 \sqrt{3} e^{2\left(\beta_{+}-\beta_{c}\right)} ;
$$

this gives an extremely useful geometrical definition of $\omega_{-}$.

Now $\omega_{-}$is an adiabatic invariant (Misner, 1969) so long as $\omega_{-}$is small. This is shown by comparing the motion of $\beta_{+}$with $\beta_{c}$. We have 
already computed $d \beta_{+} / d \Omega$; we now use Eq. (4.6) and $\beta_{c}=2 \beta_{w}$ to compute

$$
\begin{aligned}
\frac{d \beta_{c}}{d \Omega} & =1+\frac{1}{2} \frac{d}{d \Omega} \ln H \\
& \cong 1-\not \mu_{+}^{-2}(12)^{2} R_{0}^{4} e^{4 \beta_{+}-4 \Omega} \beta_{-}^{2} \\
& \cong \frac{d \beta_{+}}{d \Omega},
\end{aligned}
$$

where the equality holds to order $\left(\beta_{-}^{3}\right)$. Hence, via (4.9), $\beta_{+}-\beta_{c}$ is an adiabatic invariant and the motion can persist for a very long time.

We have now completed the study of the dynamics of these mixing modes aligned with the rotation axis, and turn to the photon propagation in these models.

\section{Null Geodesics}

Define $\tan \phi=q_{1} / q_{2}, n_{i}=q_{i} /\left(q_{k} q_{k}\right)^{1 / 2}$, and $\left(n^{0}\right)^{2}=g^{i j} n_{i} n_{j}$, where $q_{i}$ are the spatial momentum components in the $\left\{d t, \sigma^{i}\right\}$ frame. Then $q_{k} q_{k}=$ constant, and the equations for photon motion in these nontumbling type IX models read

$$
\begin{gathered}
\frac{d n_{3}}{d t}=-\frac{e^{-2 \beta+}\left(1-n_{3}^{2}\right)}{n^{0} R^{2}} \sinh 2 \sqrt{3} \beta_{-} \sin (2 \varphi+2 \phi), \\
\frac{d \phi}{d t}=-\frac{n_{3}}{n^{0} R^{2}}\left\{e^{4 \beta+}-e^{-2 \beta+}\left[\cosh 2 \sqrt{3} \beta_{-}\right.\right. \\
\left.\left.+\sinh 2 \sqrt{3} \beta_{-} \cos (2 \varphi+2 \phi)\right]\right\}
\end{gathered}
$$

(Matzner, 1971).

Clearly from Eq. (5.1), a photon with $q_{3}=q$ maintains this condition as it moves. Hence, $q_{3}=$ constant for such geodesics. It should also be noted that if $\beta_{-} \ll 1$ (i.e., if the model is mixing) then $q_{3} \simeq$ constant even if $q_{3} \neq q$.

The momentum $\boldsymbol{q}$ (a contravariant vector) is

$$
\boldsymbol{q}=q^{0} \partial_{t}+q^{i} \boldsymbol{\eta}_{i}
$$

The contravariant basis vectors $\boldsymbol{\eta}_{\alpha}$ are the dual basis of the basis $\left\{d t, \sigma^{i}\right\}$ of Eq. (1.1). We express the $\sigma^{i}$ basis of Eq. (1.1) in terms of the coordinates named $x, y, z$. The $\sigma^{i}$ are then given by the substitutions $\theta \rightarrow x, \psi \rightarrow y$, $\varphi \rightarrow z$ into Eqs. (1.10). It is straightforward to obtain the contravariant 
basis $\boldsymbol{\eta}_{i}$ (see, e.g., Misner, Wheeler, Thorne, 1970):

$$
\begin{aligned}
& \boldsymbol{\eta}_{1}=-\cos z \partial_{x}-\frac{\sin z}{\sin x}\left(\partial_{y}-\cos x \partial_{z}\right) \\
& \boldsymbol{\eta}_{2}=-\sin z \partial_{x}+\frac{\cos z}{\sin x}\left(\partial_{y}-\cos x \partial_{z}\right) \\
& \boldsymbol{\eta}_{3}=\partial_{z} .
\end{aligned}
$$

The spatial coordinate components of the momentum, $q^{i^{\prime}}$, give

$$
\frac{d x^{i^{\prime}}}{d t}=\frac{q^{i^{\prime}}}{q^{0}} \equiv\left(a^{-1}\right)_{j}^{i^{\prime}} \frac{q^{j}}{q^{0}},
$$

where $a_{j}^{i^{\prime}}$ is defined by $\boldsymbol{\eta}_{k}=a_{k}^{j^{\prime}} \partial_{j^{\prime}}$.

In a non-tumbling model, one finds

$$
\begin{aligned}
& g^{11}=e^{-2 \beta+}\left[\cosh (2 \sqrt{3}) \beta_{-}+\sinh (2 \sqrt{3}) \beta_{-} \cos 2 \varphi\right], \\
& g^{22}=e^{-2 \beta+}\left[\cosh (2 \sqrt{3}) \beta_{-}-\sinh (2 \sqrt{3}) \beta_{-} \cos 2 \varphi\right], \\
& g^{12}=e^{-2 \beta+} \sinh (2 \sqrt{3}) \beta_{-} \sin 2 \varphi, \\
& g^{33}=e^{4 \beta+}, \quad g^{31}=g^{32}=0,
\end{aligned}
$$

where the 3-axis is the rotation axis (Ryan, 1970).

Consider a photon with $q_{3}=q$ and $q_{A}=0, A \neq 3$. Via Eqs. (5.1), (5.2), and (5.5), we obtain $x^{A}=$ constant, $A=1,2$, and

$$
\frac{d z}{d t}=e_{33}^{-2 \beta} R^{-2} q_{3}\left(R^{-2} e_{33}^{-2 \beta} q_{3} q_{3}\right)^{-1 / 2},
$$

so

$$
\frac{d z}{d \Omega}=\frac{d z}{d t} \frac{d t}{d \Omega}=R^{-1} e^{2 \beta+}\left(6 R^{3} / H\right)=\frac{1}{2} \omega_{-} .
$$

We recall that $z$ is an angular coordinate; $\Delta z=4 \pi$ describes one complete circumnavigation of the universe. [See also Chitre (1970) and Doroshkevich and Novikov (1970).] One can continue the analysis as in Section II to determine the probability $P$ that the mixing will occur. We shall not repeat the discussion here.

\section{Non-aligned Mixing Axis in Non-tumbling Models}

We have so far discussed the situation when the system point is far up the channel in the $\beta_{+}$direction, where $\beta_{33}=-2 \beta_{+}$and the 3-direction is the rotation axis. This has resulted in no essential change in the Mixmaster behavior of the model; in particular, the rotation does not change 
the mixing direction since the rotation and mixing axes coincide. A distinctly different situation in the non-tumbling models has the system point moving in one of the two $V_{g}$ channels which do not correspond to the rotation direction; these two channels lie along the lines $\sqrt{3} \beta_{-}$ $= \pm \beta_{+}$. The term involving $\not_{\varphi}$ in $H^{2}$ then is

$$
\frac{3 \not_{\varphi}^{2}}{\sinh ^{2} 2 \sqrt{3} \beta_{-}} \simeq 12 \not_{\varphi}^{2} e^{-6\left(\Omega-\Omega_{0}\right)},
$$

where $\Omega_{0}$ is the instant when the channel motion began. Because of the $e^{-6 \Omega}$ dependence, we see that the $h_{\varphi}$ terms in the Hamiltonian must eventually become negligible. We shall treat the case where they are not negligible (i.e., before they become negligible) below, but here we assume the $\mu_{\varphi}$ terms are negligible so far as the dynamics is concerned. Then the non-rotating analysis of Misner (1969) obtains for the small oscillations in the channel.

The mixing direction and the rotation axis are now distinct, and because the $h_{\varphi}$ terms are not precisely zero, there is a slow rotation of the mixing direction within the 2-plane orthogonal to the 3-axis. We compute

$\frac{d \varphi}{d \Omega}=\frac{\partial H}{\partial \mu_{\varphi}} \simeq \frac{12 \mu_{\varphi} e^{-4 \sqrt{3} \beta_{-}}}{H} \simeq \frac{12{\mu_{\varphi}} e^{-6|\beta|}}{H} \simeq \frac{12{\mu_{\varphi}} e^{-6\left(\Omega-\Omega_{0}\right)}}{H}$.

If $\varphi$ changes by an arbitrarily small amount after mixing begins, the rotation will obviously have little effect on the mixing, and the nonrotating analysis of Misner (1969) will be essentially correct. As the system evolves up the channel, $d \varphi / d \Omega$ becomes small $d \varphi / d \Omega \propto R^{6}$. This corresponds roughly to $d \varphi / d t \propto R^{3}$, by Eq. (1.4). If $\varphi$ moved slowly enough that the transverse directions, orthogonal to the mixing direction, have time to equalize as the mixing direction turned, then rotation has essentially no effect on the horizon evolution. We will therefore consider the opposite case, $d \varphi / d \Omega \gg 1$. We might in fact consider $|d \varphi / d \Omega| \gg|d \phi / d \Omega|$ and $|d \varphi / d \Omega| \gg\left|d m_{3} / d \Omega\right|$, which we show shortly are smaller bounds on $|d \varphi / d \Omega|$.

In any case, one cannot anticipate spectacular results from the rotation. Ryan (1970) has considered the change in $\varphi$ during any close

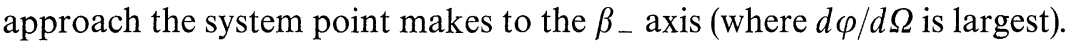
He finds $\Delta \varphi \leqq \pi / 2$ in the large anisotropy regime, if the potential $V_{g}$ is neglected. His $\Delta \varphi$ is the angle between the incident and outgoing directions in such an encounter. Only the half of the motion with $\beta_{-}$increasing can contribute to mixing. Hence, we have $(\Delta \varphi)_{\text {(mixing) }}=\pi / 4$. This is sufficient to change a pancake direction from the 1-direction to the 2-direction [see Eqs. (5.6)]. However, a fairly simple test shows that this 
motion cannot have significant effect on the mixing process because the large $d \varphi / d \Omega$ behavior does not persist sufficiently long.

Approximating both the cosine and $n_{3}$ by unity, Eq. (5.2) gives

$$
\left|\frac{d \phi}{d t}\right| \simeq \frac{R^{-1} e^{-2 \beta+} e^{2 \sqrt{3} \beta-}}{\left[e^{-2 \beta+} e^{2 \sqrt{3} \beta-}\right]^{1 / 2}},
$$

where we assume $\beta_{+}<0, \beta_{-} \gg 1$. Introducing the factor $d \Omega / d t$, we have

$$
\begin{aligned}
\left|\frac{d \phi}{d \Omega}\right| & =\frac{d \phi}{d t}\left(6 R^{3} / H\right) \\
& =0\left(\frac{6}{H} R_{0}^{2} e^{-2 \Omega} e^{-\beta_{+}+\sqrt{3} \beta-}\right) \\
& =0\left(\omega_{-} / 2\right) .
\end{aligned}
$$

The last form holds if we have corner motion so $-\beta_{+}+\sqrt{3} \beta_{-}=2|\beta|$. Recall again that typically $\omega_{-} \ll 1$. Hence, in view of Eq. (6.2), the requirement $d \varphi / d \Omega>|d \phi / d \Omega|$ demands

$$
\frac{d \varphi}{d \Omega}=12 \frac{\not_{\varphi}}{H} e^{-4 \sqrt{3} \beta-} \widetilde{S} \frac{\omega_{-}}{2},
$$

or

$$
12 \mu^{2} e^{-4 \sqrt{3} \beta-}-\left(\frac{\omega_{-}}{2} H\right)^{2} \frac{e^{4 \sqrt{3} \beta-}}{12} .
$$

The left side of Eq. (6.6) has precisely the form of the terms involving $\not_{\varphi}^{2}$ which enter the large anisotropy $H^{2}$. Now for large anisotropy, $H^{2}$ is the sum of positive terms. Hence, Eq. (6.6) is satisfied only if

$$
\frac{1}{48} \omega_{-}^{2} e^{4 \sqrt{3} \beta-}<1 .
$$

Recalling the definition [Eq. (4.8)] of the adiabatic invariant $\omega_{-}^{2}$, we see that the requirement (6.7) cannot hold indefinitely; in fact, if

$$
4 \sqrt{3} \beta_{-}=6|\beta|>4\left(\beta_{c}-|\beta|\right),
$$

the condition fails. That is, it fails when

$$
|\beta| \widetilde{2} \frac{2}{3}\left|\beta_{c 0}\right|,
$$

with $\left|\beta_{c 0}\right|$ the distance to the corner at the beginning of the corner run. Hence, the motion can persist, if $|d \beta / d \Omega| \simeq 1$, only for a time

$$
\Delta \Omega \simeq \frac{2}{3}\left|\beta_{c 0}\right| \simeq \frac{1}{3} \ln \frac{1}{\omega_{-}} \ll \frac{1}{\omega_{-}} .
$$


In contrast, Eq. (5.7) shows a time $\Delta \Omega \simeq 8 \pi / \omega_{-}$is necessary for circumnavigation.

Of course, $|\beta|$ does not obey the $|d \beta / d \Omega| \simeq 1$ law while $\mu_{\varphi}$ is nonnegligible. Instead, one has

$$
\frac{d \beta_{ \pm}}{d \Omega}=\frac{p_{ \pm}}{H},
$$

while $\varphi$ satisfies Eq. (6.5). Hence,

$$
\begin{aligned}
& \left|\frac{d \beta}{d \Omega}\right|^{2}=1-\frac{3 \mu_{\varphi}^{2}}{\sinh ^{2} 2 \sqrt{3} \beta_{-}} \frac{1}{H^{2}} \simeq 1-\frac{12 \mu_{\varphi}^{2}}{H^{2}} e^{-4 \sqrt{3} \mid \beta-1} \\
& \left|\frac{d \beta}{d \Omega}\right|^{2} \simeq 1-\frac{12 \not_{\varphi}^{2}}{H^{2}} e^{-6|\beta|} .
\end{aligned}
$$

If we let $\left|\beta_{0}\right|$ be the minimum value of $|\beta|$ (the value which makes the right-hand side vanish) and $\Omega_{0}$ the corresponding time, then it is straightforward to show $\left(\beta-\beta_{0}\right)=\frac{3}{2}\left(\Omega-\Omega_{0}\right)^{2}+0\left(\Omega-\Omega_{0}\right)^{3}$. Hence, within a very short interval, $\Delta \Omega \simeq 1,|\beta|$ will begin moving with its free velocity $|d \beta / d \Omega| \simeq 1$.

In view of the $e^{-6 \Omega}$ behavior of $d \varphi / d \Omega$, it is apparent that there is no effect from rotation on the photon trajectories after Eq. (6.7) is violated. Because the rotation is sizable for such a brief interval, it can have no large effect on the mixing.

\section{The General Rotating Mixmaster}

Return to the Lagrangian for the general type IX solution, Eq. (1.7), and suppose that all three Euler angles are changing; $\dot{\varphi} \neq 0, \dot{\theta} \neq 0, \dot{\psi} \neq 0$. We have already noted that the $\sigma^{i} / d t$ defined by Eqs. (1.8), which appear in the Lagrangian, correspond to the components of the instantaneous angular velocity in the "body frame" of an asymmetrical rotator (whose angular position is given by the Euler angles $\varphi, \theta, \psi)$. From this viewpoint, the Lagrangian (1.7), neglecting the potential terms, describes an asymmetrical rotator with time dependent principal moments of inertia:

$$
\begin{gathered}
I_{3}=2 R^{3} \sinh ^{2} 2 \sqrt{3} \beta_{-}, \quad I_{1}=2 R^{3} \sinh ^{2}\left(3 \beta_{+}-\sqrt{3} \beta_{-}\right), \\
I_{2}=2 R^{3} \sinh ^{2}\left(3 \beta_{+}+\sqrt{3} \beta_{-}\right) .
\end{gathered}
$$

The time dependent moments of inertia are also dynamical variables in the system; the potential $V_{g}$ affects only the motion of the moments of inertia. As we pointed out above, both $\not_{\psi}$ and $\not^{2}$ are constants of the 
motion of this system. Since $\partial\left(\sigma^{3} / d t\right) / \partial \dot{\varphi}=1, \partial\left(\sigma^{1} / d t\right) / \partial \varphi=-\sigma^{2} / d t$, $\partial\left(\sigma^{2} / d t\right) / \partial \varphi=\sigma^{1} / d t$, the Euler-Lagrange equation from $\mathscr{L}$ for $\varphi$ (neglecting the $T_{00}$ terms) is

$$
\frac{d}{d t}\left(I_{3} \frac{\sigma^{3}}{d t}\right)=\frac{\sigma^{1}}{d t} \frac{\sigma^{2}}{d t}\left(I_{2}-I_{1}\right)
$$

Because this expression is written in the "body frame" and since the labeling of axes is arbitrary, we have immediately the two equations obtained by cyclic interchange of indices. Let us suppose that the model is in the Mixmaster mode and has $\left|\beta_{-}\right|$small, $\left|\beta_{-}\right| \ll\left|\beta_{+}\right|$. It is then apparent that $I_{2} \simeq I_{1}$; more precisely, if $\beta_{+} \gg 1$ (as it will be if the system is moving in the direction of a corner channel with $\left|\beta_{-}\right|$small),

$$
\begin{aligned}
I_{2}-I_{1} & \simeq \frac{1}{2} R^{3}\left[e^{6 \beta+} e^{-2 \sqrt{3} \beta-}-e^{6 \beta+} e^{2 \sqrt{3} \beta-}\right] \\
& \simeq \frac{1}{2} R^{3} e^{6 \beta+}\left[-4 \sqrt{3} \beta_{-}+0\left(\beta_{-}^{2}\right)\right] .
\end{aligned}
$$

Hence, so long as $\left|\beta_{-}\right|$is small, Eq. (7.2) predicts the existence of an additional integral of the motion $h_{\varphi}=I_{3}\left(\sigma^{3} / d t\right)$. [Notice that the right side of Eq. (7.3) grows as $e^{3 \beta+} \propto e^{3 \Omega}$, but we show below that $\left(\sigma^{1} / d t\right)\left(\sigma^{2} / d t\right) \propto e^{-6 \Omega}$ so the right side of Eq. (7.2) becomes small as $\Omega$ increases.] Hence, there are three immediate integrals: $h_{\varphi}, k_{\psi}$, and $h^{2}$. Since $\beta_{\varphi}$ is an integral, the analysis of the $\beta_{-}$and $\varphi$ motion given in Section IV above for the small $\beta_{-}$case follows exactly. More interesting is the fact that now

$$
h_{\perp}^{2} \equiv \not^{2}-h_{\varphi}^{2}
$$

is a constant of the motion. Thus, $I_{1}^{2}\left(\sigma^{1} / d t\right)^{2}+I_{2}^{2}\left(\sigma^{2} / d t\right)^{2}$ is constant. Now $\beta_{-}^{2}$, as we saw from Section IV, changes very slowly $\propto e^{-2(\beta+-\Omega)}$, and hence, $I_{3} \tilde{\propto} R^{3} \propto e^{-3 \Omega}$ and $\sigma^{3} / d t \propto e^{3 \Omega}$. On the other hand, $I_{1} \sim I_{2} \propto e^{3 \Omega}$. Hence, $\left(\sigma^{1} / d t\right)^{2}+\left(\sigma^{2} / d t\right)^{2} \propto e^{-6 \Omega}$. If we suppose that $\mu_{\varphi}^{2}$ is small compared to $\not^{2}$, then during the initial part of the corner run evolution the rotation axis lies mostly in the 1-2 direction (in the "body frame"). As the system evolves, the moments of inertia $I_{1}$ and $I_{2}$ become very large, and the net rotation axis swings to align with the 3-axis. This process can result in a more effective mixing process, as the mixing is carried out in the direction the mixing axis momentarily lies in. However, the mixing will be unaffected by the rotation once the mixing axis aligns with the rotation axis. Furthermore, the decrease $\left(\sigma^{1} / d t\right)^{2}$ $+\left(\sigma^{2} / d t\right)^{2} \propto e^{-6 \Omega}$ is the same law we found for $d \varphi / d \Omega$ while investigating the non-tumbling models with distinct mixing and rotation axes. In view of the results of Section IV, we conclude that this mechanism will not have enough time to produce results significantly different from those obtained from the non-rotating Mixmaster. 


\section{Conclusion}

We have investigated both tumbling and non-tumbling rotating models and found that in no case is the rotation important long enough to affect mixing. We recall the small probability of effective mixing in non-rotating models indicated in Section II above. We must conclude, therefore, that mixing as described by the classical Einstein equations is not likely to produce large scale homogeneity in the universe.

Near the singularity, where we know the classical relations cannot hold, we hope the quantized theory may produce more likely mixing For instance, a quantum theory may produce enhanced amplitudes in configurations corresponding to mixing.

\section{References}

Arnowitt, R., Deser, S., Misner, C. W.: In: Witten, L. (Ed.): Gravitation. An Introduction to Current Research. New York: John Wiley 1962.

Chitre, D. M.: University of Maryland, Department of Physics and Astronomy Technical Report 71-040 (1970).

- preprint (1971).

Doroshkevich, A. G., Novikov, I. V.: preprint (1970).

Goldstein, H. : Classical Mechanics, Reading, Mass. : Addison-Wesley 1959.

Matzner, R.A., Shepley, L. C., Warren, J. B. : Ann. Phys. 57, 401 (1970).

- J. Math. Phys. 11, 2432 (1970).

- Ann. Phys. (N.Y.), to be published (1971).

Misner, C. W.: unpublished essay (1967).

- Astrophys. J. 151, 431 (1968).

- Phys. Rev. Letters 22, 1071 (1969a).

- Phys. Rev. 186, 1319 (1969b).

- Wheeler, J.A., Thorne, K. S.: Gravitation (prepublication edition, 1970).

Ryan, M.P., Thesis, Ph. D.: University of Maryland, Department of Physics and Astronomy (1970).

R. Matzner

D. M. Chitre

Department of Physics

University of Texas

Austin, Texas 78712

USA 